\title{
Câncer de Próstata: Adesão ao Exame Preventivo em Comunidades Carentes de Duas Capitais do Brasil
}

Prostate Cancer: Adherence to Preventive Examination in Communities in Need of Two Capitals in Brazil

Cáncer de Próstata: Adhesión al Examen Preventivo en Comunidades carentes de dos capitales de Brasil

Rozelia Vieira de Santana Na Isna ${ }^{1}$

Bidansanta $\mathrm{Na}$ Isna $^{2}$

Ana Maria Queijeiro López ${ }^{3}$

\section{Resumo}

Objetivo: Analisar a adesão de homens de áreas periféricas de Maceió-AL e Recife/PE, aos exames preventivos do Câncer de Próstata. Método: estudo descritivo, transversal, quantitativo, realizado com homens em comunidade carente de Maceió/AL e em Unidade de Saúde da Família de Recife/PE. Os dados foram coletados através de um questionário semi-estruturado. Resultados: Participaram do estudo 99 homens, sendo 50 em Maceió e 49 em
Recife. A idade média era 61,2 anos, a maioria dos participantes 37,22\% estavam na faixa etária dos 40-50 anos, $60,57 \%$ eram casados. A maior parte $(33,48 \%)$ possuía apenas o ensino fundamental incompleto e percebiam uma renda familiar de 1-2 salários mínimos e 92\% assumem sozinhos a responsabilidade financeira pela família. Quando perguntados se eles já haviam ouvido falar de câncer de próstata, 99,99\%, disseram que sim e 73,73\% deles mencionavam a mídia de televisão como agente de informação. Quando os

${ }^{1}$ Enfermeira. Pós-graduada em Programa de Saúde da Família com ênfase em Saúde Pública pelo IESC. Autora correspondente: Av. Prof. Moraes Rego, 1235, Cidade Universitária. CEP 50670-901. Recife, PE, Brasil. E-mail: rnaisna@gmail.com ${ }^{2}$ Discente do curso de graduação em Medicina pela Universidade Federal de Alagoas (UFAL). ${ }^{3}$ Professora Associada da Universidade Federal de Alagoas (UFAL).

Recebido: Abr./2018 - Aceito: Ago./2018. 
participantes foram questionados sobre o significado do câncer de próstata, apesar da maioria já terem ouvido falar sobre a doença, apenas $28 \%$ tinham uma verdadeira noção e $76 \%$ conheciam algum tipo de teste usado no diagnóstico, embora $42,42 \%$ deles já tinham sido submetidos à triagem de câncer de próstata pelo menos uma vez, dos exames para triagem o PSA isoladamente foi o mais utilizado pela maior parte dos entrevistados 15,15\%. Conclusão: Os resultados mostram que, não por desconhecimento muitos homens das comunidades estudadas ainda não aderem às práticas preventivas contra o $\mathrm{CP}$, o que torna imprescindível compreender o universo masculino, no qual se insere o estereótipo de masculinidade, propondo e direcionando estratégias educacionais assim como melhorar a oferta dos exames que auxiliem no diagnóstico e tratamento da população afetada.

\section{Descritores: Detecção Precoce de} Câncer; Fatores de Risco; Educação em Saúde.

\section{Abstract}

Objective: Analyze the adherence of men from peripheral areas of Maceió$A L$ and Recife-PE to preventive exams of Prostate Cancer (PC). Method: a descriptive, cross-sectional, quantitative approach study with 50 men (40-70 years old) from a poor community in Maceió / AL and 49 men enrolled in a Family Health Unit (USF) of an equally poor neighborhood of Recife / PE (4080 years). Data were collected through a semi-structured questionnaire. Results: 99 men participated in the study, 50 in Maceió and 49 in Recife. The average age was 61.2 years, the majority of participants $37.22 \%$ were in the age group of $40-50$ years, $60.57 \%$ were married. The majority (33.48\%) had only incomplete elementary education and perceived a family income of 1-2 minimum wages and 92\% assumed financial responsibility for the family alone. When asked if they had ever heard of prostate cancer, 99 (99.99\%) said yes and 73 (73.73\%) of them mentioned the television media as an information agent. When participants were asked about the significance of prostate cancer, although 98 (98.98\%) had already heard about the disease, only 28\% had a true notion and $76 \%$ knew some type of test used in the diagnosis, although $42(42.42 \%)$ of the interviewees had already been screened for prostate cancer at least once (mainly in the age groups 51-60 and 61-70, of the 
screening tests PSA alone was the most used by the largest of respondents 15 (15.15\%). Conclusion: The results show that, not by ignorance, many men from the studied communities still do not adhere to the preventive practices against $C P$. It is imperative to understand the masculine universe, in which the stereotype of masculinity is inserted, proposing and directing educational strategies as well as improving the offer of the tests that aid in the diagnosis and treatment of the affected population.

Descriptors: Early Detection of Cancer; Risk Factors; Health Education.

\section{Resumen}

Objetivo: Analizar la adhesión de hombres de áreas periféricas de Maceió-AL y Recife-PE a los exámenes preventivos del cáncer de próstata (CP). Método: En el presente trabajo se analizan los resultados obtenidos en el análisis de los resultados obtenidos en el análisis de los resultados obtenidos en el estudio. (40-80 años). Los datos fueron recolectados a través de un cuestionario semiestructurado. Resultados: Participaron del estudio 99 hombres, siendo 50 en Maceió y 49 en
Câncer de Próstata: Adesão ao Exame Preventivo

Recife. La edad media era 61,2 años, la mayoría de los participantes 37,22\% estaban en el grupo de edad de los 4050 años, el 60,57\% estaban casados. La mayor parte $(33,48 \%)$ poseía apenas la enseñanza fundamental incompleta $y$ percibian una renta familiar de 1-2 salarios mínimos y el 92\% asumen por sí solos la responsabilidad financiera por la familia. Cuando se les preguntó si ya habian oído hablar de cáncer de próstata, 99 (99,99\%), dijeron que sí y $73(73,73 \%)$ de ellos mencionaban a los medios de televisión como agente de información. Cuando los participantes fueron cuestionados sobre el significado del cáncer de próstata, a pesar de que 98 (98,98\%) ya habian oído hablar de la enfermedad, sólo el 28\% tenía una verdadera noción y el $76 \%$ conocía algún tipo de prueba utilizado en el diagnóstico, aunque $(42,42 \%)$ de los entrevistados ya han sido sometidos a la selección de cáncer de próstata por lo menos una vez (principalmente en los grupos de edad 51-60 y 61-70, de los exámenes para clasificación el PSA aisladamente fue el más utilizado por la mayor parte de los entrevistados 15 (15,15\%). Conclusión: Los resultados muestran que, no por desconocimiento muchos hombres de las comunidades estudiadas todavía no se adhieren a las prácticas preventivas contra el CP. Es 
imprescindible comprender el universo masculino, en el cual se inserta el estereotipo de masculinidad, proponiendo y dirigiendo estrategias educativas así como mejorar la oferta de los exámenes que auxilien en el diagnóstico y tratamiento de la población afectada.

Descriptores: Detección Precóz del Cáncer; Factores de Riesgo;

\section{Educación en Salud.}

\section{Introdução}

Os Cânceres configuram uma das principais causas de morbimortalidade em todo o mundo, com aproximadamente 14 milhões de novos casos e 8,2 milhões de mortes relacionadas ao câncer em 2012. O número de novos casos deverá aumentar cerca de $(70 \%)$ nas próximas 2 décadas. Entre os homens, os 5 locais mais comuns de câncer diagnosticados em 2012 foram câncer de pulmão, próstata, colorretal, estômago e fígado ${ }^{(1)}$.

A próstata é uma glândula exócrina, anexa do aparelho genital masculino que está situada abaixo da bexiga e envolve parte inicial da uretra. Tem por finalidade armazenar e secretar um fluido alcalino que, em conjunto com os espermatozóides, constitui a maior parte do sêmen. Essa glândula está sujeita a uma série de patologias, mas é, sobretudo, conhecida pelos tumores de que é sede, ou seja, o Câncer de Próstata $(\mathrm{CaP})^{(2)}$.

Pacientes com $\mathrm{CaP}$ em sua fase inicial, passível de tratamento curativo, não desenvolvem, qualquer sinal ou sintoma relacionado à neoplasia. Os sintomas aparecerão na doença localmente avançada na fase metastática ${ }^{(3)}$. Dificuldade ao urinar, jato urinário fraco e sensação de não esvaziar completamente a bexiga, aumentando as chances de cura ou mesmo evitada quando o processo é diagnosticado e tratado com precocidade permitindo um tratamento menos agressivo ou mutilante ${ }^{(4)}$.

O Câncer de Próstata é o mais comum entre homens em todas as regiões do Brasil, ficando atrás apenas do câncer de pele não melanoma ${ }^{(5)}$. Uma estimativa do Instituto Nacional do Câncer (INCA) revela o aparecimento de 61.200 novos casos de câncer de próstata no biênio 2016/2017 e 13.772 mortes pela doença ${ }^{(6)}$.

A idade é o único fator de risco bem estabelecido para o desenvolvimento da doença prostática, com aproximadamente $62 \%$ dos casos diagnosticados no mundo ocorrendo em homens com idade de 65 anos ou mais. 
Com o aumento da expectativa de vida mundial, é esperado que o número de casos novos aumente ${ }^{(6)}$. Aspectos étnicos e hábitos alimentares também são considerados como fatores de risco (7) A história familiar também apresenta importância. Se um parente de primeiro grau tem a doença, o risco é, no mínimo, duas vezes maior do indivíduo ter $\mathrm{CaP}$. Se dois ou mais indivíduos da mesma família são afetados, o risco aumenta em cinco a 11 vezes. Porém, a hereditariedade não parece ser fator prognóstico importante ou influenciar negativamente a mortalidade relacionada ao $\mathrm{CaP}^{(3)}$.

O Nordeste brasileiro é a terceira região do país com maior incidência desta doença. Individualizando o estado de Pernambuco, em 2016, os novos casos estimados dessa neoplasia estão na faixa de 2.750 novos casos por 100.000 habitantes. Em Alagoas, tiveram em 2016 cerca de 560 novos casos por 100.000 habitantes ${ }^{(8,9)}$.

A alta taxa de câncer de próstata pode estar relacionada ao fato da dificuldade dos homens em acessar a atenção primária, seja por desconhecimento, preconceito ou falta de recursos médico local, prejudica a realização do rastreamento do $\mathrm{CaP}$ e, consequentemente, impossibilita a detecção de tumores em fase inicial, prejudicando o diagnóstico e tratamento precoce ${ }^{(10)}$. Portanto, é importante investir em medidas para incentivar a adesão às formas utilizadas para o diagnóstico da doença.

Diante desse contexto, o presente estudo foi desenvolvido com o objetivo de analisar a adesão de homens aos exames diagnósticos do Câncer de Próstata em áreas periféricas de duas capitais do nordeste brasileiro.

\section{Método}

Trata-se de uma pesquisa de caráter descritivo, transversal, de abordagem quantitativa, quantificando a opinião da amostra sobre o assunto, que é exploratório porque busca descrever a realidade dos usuários de uma Unidade de Saúde da Família (USF) em Recife (Pernambuco-Brasil), bem como de uma comunidade carente da vila de Benedito Bentes, em Maceió (Alagoas, Brasil). Levadas em consideração as recomendações preconizadas de acordo com a Resolução n ${ }^{\circ}$ 466/12 do Conselho Nacional de Saúde sobre pesquisas com seres humanos. A pesquisa foi aprovada pelo Comitê de Ética em Pesquisa do Hospital do Câncer de Pernambuco, dirigindo-se à CAEE 31811513.1.0000.5205. 
Os participantes no Recife foram 49 homens com idade entre 40 e 80 anos, que procuraram os serviços da USF, e em Maceió havia 50 homens com idade entre 40-70 anos, que utilizavam o terminal de ônibus na Vila Benedito Bentes e estavam dentro dos critérios de inclusão . A coleta de dados em Recife ocorreu em agosto e setembro de 2014, e em Maceió foi realizada em setembro e outubro de 2016, utilizando um instrumento composto por questões abertas e fechadas, a serem respondidas pelos sujeitos da pesquisa, exceto aqueles que solicitaram ao pesquisador ler $\mathrm{o}$ documento para eles.

As perguntas consideram os dados de identificação do usuário (como nome, idade, nível educacional, profíssão e tempo de execução, estado civil, renda familiar, etc.) e perguntas orientadoras (como: "você já ouviu falar sobre câncer de próstata?", qual é a fonte de informação usada por você sobre esse assunto? "," você sabe o que é câncer de próstata? "," você sabe quais são os testes diagnósticos para o câncer de próstata? "," você já foi submetido a exames de diagnóstico, próstata câncer? ", e" você conhece alguém em sua Família que já foi diagnosticado com câncer de próstata? "). Para análise dos dados obtidos nas questões foi utilizada estatistica descritiva. $\mathrm{O}$ banco de dados foi elaborado através do programa Microsoft Excel ${ }^{\mathrm{R}}$. Os dados são apresentados sob a forma de frequências absoluta e relativa, finalmente, os resultados foram discutidos com base na literatura sobre o assunto.

\section{Resultados}

Participaram do estudo 99 homens, sendo 50 em Maceió e 49 em Recife. Os resultados obtidos através da pesquisa mostram que a idade média era 61,2 anos, a maioria dos participantes $37,22 \%$ estavam na faixa etária dos 40-50 anos, 60,57\% eram casados. Desse montante a maior parte $(33,48 \%)$ possuía apenas o ensino fundamental incompleto e percebiam uma renda familiar de 1-2 salários mínimos e $92 \%$ assumem sozinhos a responsabilidade pela família (18\% com 2 membros, 24\% com 3 membros, 28\% com 4 membros, $30 \%$ com mais de 5 membros). As profissões exercidas variaram de jardineiro, pedreiro, fazendeiro, motorista de veículos grandes ou motocicletas, segurança, garçom, serralheiro, pintor, polidor, mecânico, estibordo, açougueiro, vendedores (vendedores ambulantes ou não), corretor de imóveis, secretário da 
escola e servidor público (função não

declarada). (Tabela 1).

Tabela 1 - Condições Sociodemográficas

Variável

dade (anos)

40 a 50

51 a 60

61 a 70

71 a 80

Escolaridade

Não alfabetizado

Ensino Fundamental Incompleto

Ensino Fundamental Completo

Ensino Médio Incompleto

Ensino Médio Completo

Ensino Superior Incompleto

Ensino Superior Completo

Estado Conjugal

Casado

Solteiro

União Estável

Separado ou Divorciado

Renda Familiar per capita (salários mínimos)
2652,00

$13 \quad 26,00$

$11 \quad 22,00$

$0 \quad 0,00$

$9 \quad 18,00$

$9 \quad 18,00$

36,00

$8 \quad 16,00$

$11 \quad 22,00$

$6 \quad 12,00$

48,00

3264,00

$9 \quad 18,00$

12,00

$8 \quad 16,00$

$5 \quad 10,00$

$30 \quad 60,00$

$6 \quad 12,00$

$4 \quad 8,00$

$5 \quad 10,00$
Recife

n (49) \%

Abaixo de 1

1 a 2

2 a 3

3 a 4

Acima de 5

$\begin{array}{rrrr}5 & 10,00 & 6 & 12,24 \\ 30 & 60,00 & 34 & 69,38 \\ 6 & 12,00 & 7 & 14,28 \\ 4 & 8,00 & 2 & 4,08 \\ 5 & 10,00 & 0 & 0,00\end{array}$


Em relação aos problemas de saúde, $\quad 29,29 \%$ dos entrevistados afirmam que praticaram atividade física - discriminadas apenas como caminhadas ou práticas de futebol, e $57,57 \%$ declararam que nunca fumaram (mais na faixa etária 40-50), embora $55,55 \%$ dizerem que frequentemente bebem álcool (a maioria na faixa etária 40-50 ou 61-70). Quando perguntados se eles já haviam ouvido falar de câncer de próstata, 99,99\%, disseram que sim e $73,73 \%$ deles mencionaram a mídia de televisão como agente de informação.

Tabela 2 - Informações dos Entrevistados Acerca do Conhecimento Sobre Câncer de Próstata

\begin{tabular}{|c|c|c|c|c|}
\hline \multirow[t]{2}{*}{ Respostas dos sujeitos } & \multicolumn{2}{|c|}{ Maceió } & \multicolumn{2}{|c|}{ Recife } \\
\hline & $\mathrm{n}^{\mathrm{o}}(50)$ & $\%$ & $\mathrm{n}^{\mathrm{o}}(49)$ & $\%$ \\
\hline \multicolumn{5}{|l|}{ Já ouviu falar sobre câncer de próstata } \\
\hline $\operatorname{Sim}$ & 50 & 100 & 48 & 97,95 \\
\hline Não & 0 & 0 & 1 & 2,05 \\
\hline \multicolumn{5}{|l|}{ Agente de informação* } \\
\hline Televisão & 38 & 76 & 35 & 71,42 \\
\hline Rádio & 2 & 4 & 7 & 14,28 \\
\hline Família/amigos & 15 & 30 & 21 & 42,85 \\
\hline Médico & 12 & 24 & 5 & 10,20 \\
\hline Enfermeiro & 3 & 6 & 0 & 0,00 \\
\hline Posto de Saúde & 6 & 12 & 6 & 12,24 \\
\hline Outros & 2 & 4 & 4 & 8,16 \\
\hline \multicolumn{5}{|l|}{ Realização dos Exames } \\
\hline Não realizaram exames & 29 & 58 & 28 & 57,14 \\
\hline Já realizaram exames & 21 & 42 & 21 & 42,86 \\
\hline \multicolumn{5}{|l|}{ Exames específicos* } \\
\hline Somente PSA & 7 & 14 & 8 & 16,32 \\
\hline Somente Toque retal & 2 & 4 & 5 & 10,20 \\
\hline Ultrassonografia & 7 & 14 & 7 & 14,28 \\
\hline PSA + Toque retal & 0 & 0 & 3 & 6,12 \\
\hline PSA + Ultrassonografia & 0 & 0 & 2 & 4,08 \\
\hline PSA + Ultrassonografia + Toque retal & 5 & 10 & 3 & 6,12 \\
\hline \multicolumn{5}{|l|}{ Casos de Câncer de Próstata na Família } \\
\hline Não sabem & 2 & 4 & 33 & 67,34 \\
\hline Existem (pai, tios, irmãos, primos) & 11 & 22 & 7 & 14,28 \\
\hline Não existem & 37 & 74 & 9 & 18,36 \\
\hline
\end{tabular}

*Pergunta com opção de respostas múltiplas.

Quando os participantes foram questionados sobre o significado do câncer de próstata, apesar de 98,98\% já tivessem ouvido falar sobre a doença, apenas 28\% tinham uma verdadeira noção, a maioria na faixa etária dos $40-$
50 anos e $76 \%$ conheciam algum tipo de teste usado no diagnóstico (a maioria deles com mais de 50 anos), embora $42,42 \%$ dos entrevistados já tenham sido submetidos à triagem de câncer de próstata pelo menos uma vez 
(principalmente nos grupos de idade 5160 e 61-70, dos exames para triagem o PSA isoladamente foi o mais utilizado pela maior parte dos entrevistados
$15,15 \%$, já a combinação do PSA+ TR foi realizada por apenas 3,03\% dos entrevistados.

\section{Tabela 3 - Motivos da Não Procura da Prevenção do Câncer de Próstata}

Respostas dos sujeitos
Dificuldade para marcar consultas/exames
Vergonha
Não tem tempo
Não estarem doentes
Não sabem da existência dos exames
Não serem velhos para se preocupar com a doença
Medo
Descuido
Outros
Total

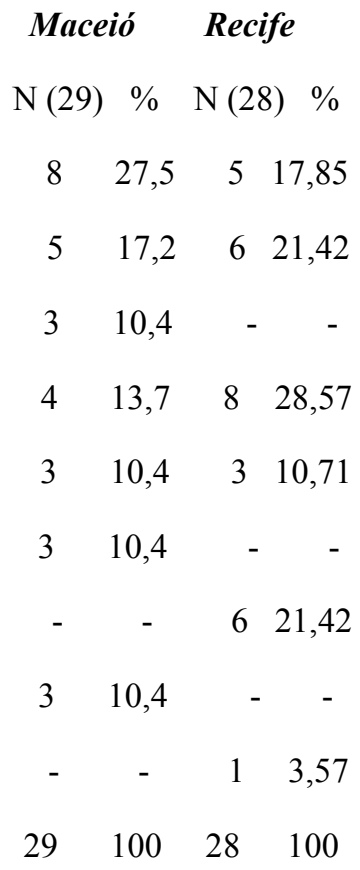

No que diz respeito ao conhecimento dos casos de câncer de próstata na família, apenas 18,18\% relataram conhecer casos de pais, tios, irmãos ou primos. (Tabela 2).

Na população estudada os 57,57\% homens que afirmaram nunca terem feito nenhum tipo de teste de diagnóstico para a doença aqui discutida e justificaram sua ausência por "dificuldades para marcar consultas/exames" seguido de" não estarem doentes" e "vergonha". (Tabela 3).

\section{Discussão}

A faixa etária tem sido considerada um importante fator de risco, dependendo do tipo de neoplasia, o que pode representar o efeito acumulativo no decorrer da vida aos agentes causadores do câncer e a idade é um fator bem estabelecido, como no caso do $\mathrm{CaP}$, no qual aproximadamente $62 \%$ diagnosticado no mundo são em idade superior a 65 anos ${ }^{(11)}$.

Quanto à escolaridade, dados desta pesquisa corroboram um estudo 
realizado em um centro de alta complexidade de tratamento para câncer na Região Noroeste do Estado do Rio Grande do Sul, Brasil, que objetivou avaliar o conhecimento acerca da doença oncológica e práticas de cuidado com a saúde, em que $68,3 \%$ da população possuía Ensino Fundamental incompleto. Nesse mesmo estudo os pesquisadores relatam que 63,1 de sua amostra possuem renda familiar de 1 a 2 salários mínimos ${ }^{(11)}$. Em relação ao estado civil, Weiss ${ }^{(12)}$ em sua pesquisa registra que $79,8 \%$ dos homens eram casados o que corrobora com os dados deste estudo.

$\mathrm{O}$ estudo constatou que em relação à fonte de informação, a impressa televisiva foi citada por maior parte dos entrevistados, ficando os profissionais de saúde com os menores índices de citações como meio de informação, sendo que esse dado está em desacordo com outro estudo realizado, onde as informações acerca da doença e seu diagnostico são pouco difundidas por esses profissionais ${ }^{(13)}$.

Quanto ao conhecimento sobre a doença Czorny ${ }^{(14)}$, em sua pesquisa realizada em uma Unidade Básica de Saúde em São Paulo, com 150 homens relataram que o PSA isoladamente foi o exame que apresentou maior frequência, $56 \%$ dos seus entrevistados. Outro estudo realizado em uma unidade de saúde no Rio de Janeiro, com 61 pacientes revelou que a combinação do PSA+TR foi realizada por maior parte dos participantes $28(45,9 \%)^{(15)}$.

Vieira ${ }^{(16)}$ relata em seu estudo com objetivo de revelar sentimentos, pensamentos e ações dos homens, relata que a maioria dos homens justifica a não adesão ao exame por não se sentir à vontade, preconceito ou terem medo de exames.

\section{Conclusão}

Torna-se necessário propor e implementar estratégias educacionais assim como melhorar a oferta dos exames de triagem para o $\mathrm{CaP}$. Os profissionais de saúde, especialmente os médicos e enfermeiros devem estar atentos para as particularidades dos homens com intuito de incentivá-los as práticas de saúde para a promoção na melhoria da qualidade de vida desses indivíduos, proporcionando um ambiente e uma situação adequada que venha gerar uma consciência sobre o cuidado, a prevenção e as conseqüências que um $\mathrm{CaP}$ diagnosticado tardiamente possa trazer. $\mathrm{O}$ incentivo ao diálogo entre a população masculina e os profissionais de saúde contemplando as perspectivas 
emocionais, comportamental, culturais e sociais possibilitará uma reflexão quanto aos cuidados com a saúde e a quebra de tabus e a busca pelos serviços de saúde por parte dos usuários.

\section{Referências}

1. WHO. World Health Organization - Cancer. Geneva: WHO; 2018. Disponível em: http://www.who.int/c ancer/en/.

2. Smeltzer SC \& Bare BG. Brunner \& Suddarth Tratado de Enfermagem Médico-Cirúrgica. Rio de Janeiro: Guanabara Koogan; 2016;10(4): 11634.

3. Damião R, Figueiredo RT, Dornas MC, Lima DS, Korschorke MAB. Câncer de próstata. Med HUPE-UERJ. Saúde (Rio de Janeiro) [periódico na Internet]. 2015 [citado 2017 nov. 25];14(1):80-6. Disponível em: http://www.e-publicacoes.uerj.br/index .php/revistahupe/article/view/17931/13 463.

4. Maia LFS. Câncer de próstata: preconceitos, masculinidade e a qualidade de vida. Enfermagem (São Paulo) [periódico na Internet]. 2012 [citado 2087 jan. 22]; 2(6):16-0. Disponível em: https://www.recien. com.br/index.php/Recien/article/view/ 42 .

5. Modesto AADA, de Lima RLB, D'Angelis AC, Augusto DK. Um novembro não tão azul: debatendo rastreamento de câncer de câncer de próstata e sáude do Homem. Saúde e Educação (Botucatu) [periódico na Internet]. 2018 [citado 2018 jun. 19]; 22( 64 ): 251-62. Disponível em: http://dx.doi.org/10.1590/1807-576220 16.0288 .
6. Instituto Nacional de Câncer José Alencar Gomes da Silva. Coordenação de Prevenção e Vigilância Estimativa 2016: incidência de câncer no Brasil / Instituto Nacional de Câncer José Alencar Gomes da Silva. Rio de Janeiro: INCA; 2015.

7. Fernandes AAC, Leandro FS, Oliveira GS, Araújo CLO. Sexualidade em homens com câncer de próstata. Enfermagem (Paraíba) [periódico na Internet]. 2014 [citado 2017 dez. 12]; 1(6):113-22. Disponível em: http://fate a.br/seer3/index.php/REENVAP/articl e/download/57/46/.

8. Brasil. Instituto Brasileiro de Geografia e Estatística (IBGE). Brasília. IBGE; 2016.

9. Pernambuco. Governo do Estado. Recife (PE). Pernambuco. Prefeitura; 2015.

10. Paiva EP, Motta MCS, Griep,RH. Barreiras em relação aos exames de rastreamento do câncer de próstata. Enfermagem (Juiz de Fora) [periódico na Internet]. 2011 [citado 2017 ago. 20]; 19(1): 73-0. Disponível em: http://www.scielo.br/pdf/rlae/v19n1/pt _11.pdf.

11. Herr GH, Kolankiewicz ACB, Berlezi EM, Gomes JS, Magnago TSBS, Rosanelli CP, Loro MM. Avaliação de conhecimentos acerca da doença oncológica e práticas de cuidado com a saúde. Cancerologia (Brasília) [periódico na Internet]. 2013 [citado 2017 set. 22]; 59(1):33-44. Disponível em: http://www1.inca.gov. br/rbc/n_59/v01/pdf/06-avaliacao-de-c onhecimentos-acerca-da-doenca-oncol ogica-e-praticas-de-cuidado-com-a-sau de.pdf. 
12. Weiss EC, Rosanelli CLSP, Loro MM, Herr GEG, Kolankiewicz ACB. Doença oncológica - conhecimento e práticas de cuidado de homens em tratamento. Saúde (Rio Grande do Sul) [periódico na Internet]. 2015 [citado 2017 abr. 15]; 15(28):67-75. Disponível em: https://www.revistas. unijui.edu.br/index.php/contextoesaude /article/view/3048/3691.

13. Oliveira PSD, Rocha RMB, Aguiar VMSN, Barbosa HA, Torres JDPRV. Prevenir para não ter: avaliando o conhecimento dos homens sobre prevenção do câncer de próstata. Enfermagem (Pernambuco) [periódico na internet]. 2017 [citado 2017 jan. 10]; 11(1): 368-73. Disponível em: https://periódicos.ufpe.br/revistas/revis taenfermagem/article/viewFile/11917/ 14409.

14. Czorny RCN, Gazetta CE, Pinto $\mathrm{MH}$, Ribeiro RCHM, Beretta D, Rodrigues CC. Perfil do usuário homem atendido em uma unidade básica de saúde da família. Enfermagem (Pernambuco) [periódico na internet]. 2017 [citado 2017 abr. 1]; 11(4):1624-31. Disponível em: https://periodicos.ufpe.br/revistas/revis taenfermagem/article/download/15231/ 17999.

15. Silva ABM, Costa, CMA, Spíndola T, Ramos RCA, Martins ERC, Francisco MTR. Conhecimentos e práticas sobre prevenção do câncer de próstata: uma contribuição para a enfermagem. Enfermagem (Rio de Janeiro) [periódico na Internet]. 2013 [citado 2018 mai. 12]; 21:785-91. Disponível em: http://www.facenf. uerj.br/v21esp2/v21e2a15.pdf.
16. Vieira CG, Araújo WS, Vargas DRM. O homem e o câncer de próstata: prováveis reações diante de um possível diagnóstico. Enfermagem (Araguaína) [periódico na Internet]. 2012 [citado 2017 out. 17]; 5(1):15-2. Disponível em: http://acimarmarialva. com.br/admin/arquivo_publicacoes/13 80815497_cancer_de_prostata.pdf. 